Review Article

\title{
Mechanisms and role of microRNA deregulation in cancer onset and progression
}

Edenir Inês Palmero ${ }^{1}$, Silvana Gisele P de Campos ${ }^{1}$, Marcelo Campos ${ }^{1}$, Naiara C Nogueira de Souza ${ }^{1}$, Ismael Dale C. Guerreiro ${ }^{1,2}$, Andre L. Carvalho ${ }^{1}$ and Marcia Maria C. Marques ${ }^{1}$

${ }^{1}$ Centro de Pesquisa de Oncologia Molecular, Hospital de Câncer de Barretos/Fundação Pio XII, Barretos, SP, Brazil.

${ }^{2}$ Departamento de Ginecologia, Universidade Federal de São Paulo, São Paulo, SP, Brazil.

\begin{abstract}
MicroRNAs are key regulators of various fundamental biological processes and, although representing only a small portion of the genome, they regulate a much larger population of target genes. Mature microRNAs (miRNAs) are single-stranded RNA molecules of 20-23 nucleotide (nt) length that control gene expression in many cellular processes. These molecules typically reduce the stability of mRNAs, including those of genes that mediate processes in tumorigenesis, such as inflammation, cell cycle regulation, stress response, differentiation, apoptosis and invasion. MicroRNA targeting is mostly achieved through specific base-pairing interactions between the 5' end ('seed' region) of the miRNA and sites within coding and untranslated regions (UTRs) of mRNAs; target sites in the 3' UTR diminish mRNA stability. Since miRNAs frequently target hundreds of mRNAs, miRNA regulatory pathways are complex. Calin and Croce were the first to demonstrate a connection between microRNAs and increased risk of developing cancer, and meanwhile the role of microRNAs in carcinogenesis has definitively been evidenced. It needs to be considered that the complex mechanism of gene regulation by microRNAs is profoundly influenced by variation in gene sequence (polymorphisms) of the target sites. Thus, individual variability could cause patients to present differential risks regarding several diseases. Aiming to provide a critical overview of miRNA dysregulation in cancer, this article reviews the growing number of studies that have shown the importance of these small molecules and how these microRNAs can affect or be affected by genetic and epigenetic mechanisms.
\end{abstract}

Key words: microRNA, cancer, genetic alteration, epigenetic alteration, therapeutic application.

Received: April 6, 2011; Accepted: June 15, 2011.

\section{MicroRNAs: Characterization and Biogenesis}

MicroRNAs (miRNAs) are a group of small RNAs, with around 19 to 25 nucleotides, resulting from cleavage of larger non-coding RNAs. They act as post-transcriptional regulators of gene expression, both in plants and in animals (Bartel, 2004). In 1993, the first miRNA, named lin-4 (lineage-deficient-4), was discovered in Caenorhabditis elegans, and was found to be associated with regulation of larval development. The second miRNA, let-7, was discovered in 2000, also in Caenorhabditis elegans. The fundamental discovery of the regulatory processes governed by miRNAs was a major stimulus for the scientific community and led to a large number of important research on miRNAs (Ambros, 2003). Over the last seven years, more than 4500 of miRNAs have been identified in the genomes of nematodes, flies, plants, viruses and humans. It has been esti-

Send correspondence to Márcia Maria Chiquitelli Marques Silveira. Centro de Pesquisa de Oncologia Molecular, Hospital de Câncer de Barretos, Rua Antenor Duarte Vilela 1331, 14784-400 Barretos, SP, Brazil. E-mail: mmcmsilveira@gmail.com. mated that more than 1000 miRNAs exist in the human genome. Estimates suggest that miRNA regulatory processes may modulate the expression of 1 to $4 \%$ of human genes, thus making miRNAs one of the largest classes of genomic regulators (Calin et al., 2004).

In mammals, miRNAs have been associated with diverse molecular pathways including regulation of proliferation, apoptosis, differentiation, cell cycle regulation hematopoiesis, and many more cellular processes. Recent studies have emphasized the importance of understanding the mechanism of mRNA regulation by studying changes in miRNA expression in a variety of human pathological conditions, including cancers (Vanderboom et al., 2008). Given their importance in development, it was to be expected that miRNAs would also have a significant role in tumorigenesis. Since their discovery close to 3000 publications, including over 700 reviews, documented associations between miRNAs and cancer, (Garofalo and Croce, 2010; Medina and Slack, 2008).

The miRNAs of different organisms have very similar overall patterns of three-dimensional structure, even 
though they frequently differ significantly in their precise cellular functions (Miyoshi et al., 2010). The biogenesis of miRNAs begins with their transcription by RNA polymerase II, generating a long primary transcript (pri-miRNA) with a cap5' start and a polyA tail. This transcript has a secondary hairpin-shaped structure and while still in the nucleus it is cleaved by RNaseIII and Drosha and their cofactor DGCR8 (Di George Syndrome critical region gene 8 ), thus generating a precursor molecule of approximately 70 nucleotides. The pre-miRNA is then quickly transported to the cytoplasm via exportina-5 (Exp5), a nuclear exportation protein that makes use of Ran-GTP as a cofactor.

Once in the cytoplasm, the pre-miRNA is processed by RNase III and Dicer, thereby generating a double strand of miRNA, of approximately 22 nucleotides in length. This product binds to the RISC complex (an induction complex for RNA silencing) and directs sequence-specific cleavage of target mRNAs Altyernatively, the miRNA may repress translation by remaining bound to the mRNA, thereby impeding its translation (Figure 1). Such transcriptional repression has been shown to play an important role in regulating growth and differentiation (Ambros, 2003; Medina and Slack, 2008), and abnormal expression of components of the miRNA regulatory complex has been correlated with different human tumors (discussed below).

Post-transcriptional regulation by miRNAs at the untranslated 3' region depends on the extent of sequence homology with the target mRNA ("templates"), and this may influence either inhibition of translation of the template, or facilitate degradation of the mRNA target transcript. Imperfect matching with mRNA leads to variable inhibition of translation of the target, and this mechanism is the main mode of action of miRNAs in mammals. The observation that miRNAs are typically short sequences that can act without the need for complete matching means that

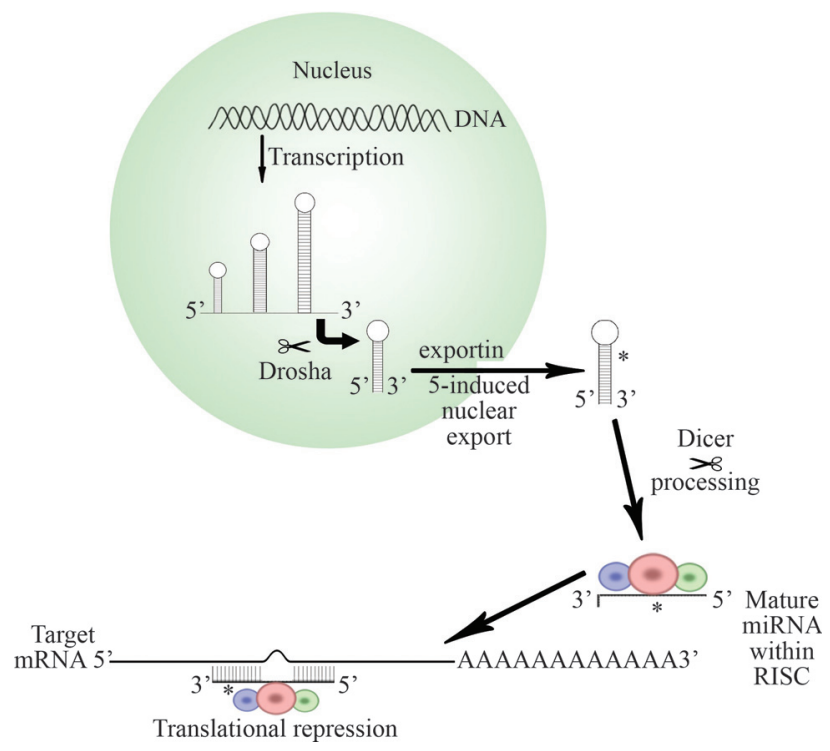

Figure 1 - The biogenesis and function of miRNAs. a single miRNA can regulate many target mRNAs. The converse also holds, so that individual miRNAs may cooperate and collectively control one single mRNA target (Calin et al., 2004).

The molecular biology of miRNAs and how they de facto act in organisms is still only beginning to be understood. There is a growing number of studies that reveal the importance of these small RNAs in diverse biological processes. Moreover, through the overall regulation of cellular gene expression and associations with different functional pathways it has become clear that miRNAs may be involved in various human diseases.

\section{Regulation of Gene Expression Through MicroRNAs and Implications for Cancer}

The current challenge is to identify target transcripts and pathways that are regulated by miRNAs. Studies have shown that a single miRISC complex may bind to more than 200 target genes, which may have a variety of functions, such as transcription factors, receptors and transporters. Thus, miRNAs can control the expression of almost one third of the human mRNA population, and deletions or modifications in this expression may contribute towards a variety of diseases, as well as disrupt pathways of fundamental importance in neoplasia (Miyoshi et al., 2010).

Cancer is a complex genetic disease involving changes in structures and gene expression. For almost three decades, carcinogenesis has been primarily attributed to abnormalities in oncogenes and tumor-suppressing genes. It is now recognized that miRNA also have a primary role in cancer onset and progression. Oncomir is the term used to describe an miRNA involved in cancer. Such miRNAs were initially linked to tumorigenesis due to their proximity to chromosomal breakpoints (Calin et al., 2004b) and their dysregulated expression levels in many malignancies $(\mathrm{Ca}-$ lin et al., 2004a). Abnormal gene expression by miRNAs has been correlated with several types of tumors, and such genes may function as oncogenes or tumor-suppressing genes (Figure 2). In humans, $50 \%$ of the miRNA genes are located at genomic sites associated with cancer-specific chromosomal rearrangements. A prime example are the genes miR-15 and miR-16, which are located on chromosome $13 \mathrm{q} 14$, a region that is deleted in more than half of the cases of chronic lymphocytic leukemia and B-cell leukemia (Calin et al., 2002). It has also been reported that miR-15a and miR-16-1 negatively regulate the expression of $B C L 2$, an anti-apoptotic oncogene that is generally overexpressed in a variety of tumors, including leukemias and lymphomas (Calin et al., 2008). These findings suggest that miR-15a and miR-16 may act as tumor-suppressing genes in human cancer.

The miRNAs that code for the let-7 family were the first group of oncomirs identified. These regulate the expression of oncogenes, and specifically the $R A S$ genes. Mutations of the RAS oncogene are present in around 25\%- 


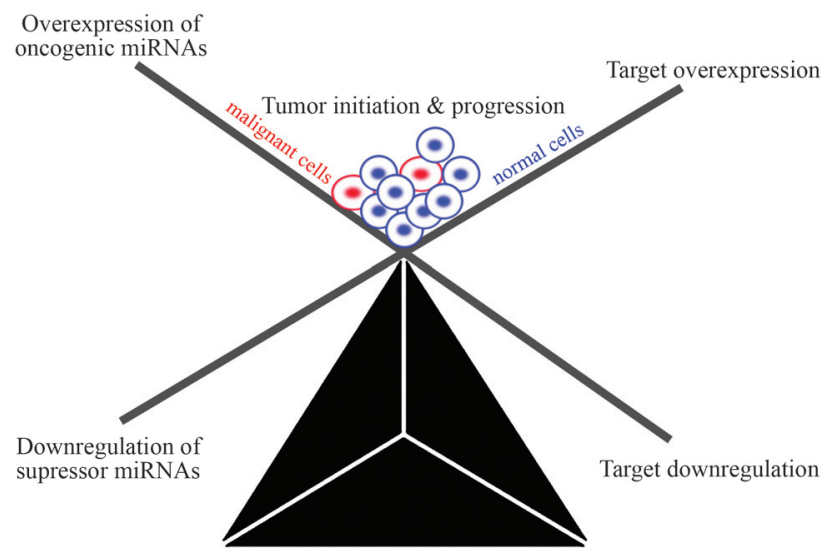

Figure 2 - MicroRNAs (miRNAs) as tumour suppressors and oncogenes. Downregulation or loss of miRNAs with tumour suppressor function may increase translation of oncogenes and hence formation of excess oncogenic proteins, leading to tumour formation. In contrast, upregulation of oncogenic miRNAs may block tumour suppressor genes and also lead to tumour formation.

$30 \%$ of all human tumors, and overexpression of the $R A S$ oncogene is very common in lung cancer cases. Ras proteins are membrane proteins that regulate cell growth and differentiation through MAP kinase signaling. In vitro experiments on a pulmonary adenoma cell lineage showed that let-7 was able to inhibit cell proliferation though Ras, inferring that let-7 may function as a tumor suppressor in this context (Johnson et al., 2005). Thus, the let-7 miRNA that regulates the expression of the Ras protein is also able to indirectly alter the cell proliferation rate through its downstream MAP signaling cascade.

The strongest evidence for an association between miRNAs and cancer was demonstrated by a sequence of three concurrent studies published in Nature in June 2005 (He et al., 2005; Lu et al., 2005; O’Donnell et al., 2005). The MYC oncogene, which codes for a transcription factor and functions as a cell growth regulator to induce proliferation and apoptosis, frequently appears mutated or amplified in human tumors. These authors reported that the miRNA group miR-17-92 (composed of seven miRNAs: miR-17-5p, miR-17-3p, miR-18, miR-19a, miR-20, miR-19b-1 and miR-92-1) was deregulated leading to increased expression of $M Y C$, culminating with development of B-cell neoplasia.

Recent studies have demonstrated that the expression levels of miR-143 and miR-145 are significantly lower in colorectal tumors, thus suggesting that these miRNAs act as potential tumor suppressors (Arndt et al., 2009). Several classes of deregulated miRNAs have also been shown to be differentially expressed in breast cancer, compared with healthy breast tissue (Volinia et al., 2006). Moreover, the expression signatures of informative miRNA subsets have enabled better molecular classification than mRNA expression profiles in several types of human cancer (Lu et al., 2005).

Among the miRNAs differentially expressed in breast cancer, miR-10b, miR-125, miR-145, miR-21 and miR-155 have consistently presented the highest degree of deregulation. Downregulation of miR-10b, miR-125b and miR-145 and upregulation of miR-21 and miR-155 suggest that these miRNAs may play an important role as tumor-suppressors or oncogenes (Blenkiron et al., 2007). In particular, the miR-145 miRNA is progressively downregulated when passing from healthy breast tissue to breast cancer with high cell proliferation rates. Similarly, but in the opposite direction, the expression of miR-21 is progressively upregulated when comparing normal breast tissue with breast cancer at advanced stages. Thus, the deregulation of these miRNAs may affect molecular events that are critical for tumor progression (Yang et al., 2008).

Some specific miRNAs have also been associated with tumor invasion and metastasis in breast cancer. For example, the level of miR-10b expression in primary breast carcinomas has been correlated with clinical progression of the disease (Ma et al., 2007). Recently it was also observed that the expression of miR-7, miR-128a, miR-210 and miR-516-3p was associated with aggressiveness in cases that were positive for estrogen-receptor tumors and negative for lymph node tumors (Foekens et al., 2008).

In recent years, studies on miRNAs, especially on a large scale using microarrays, have provided a more comprehensive picture on the role of abnormal miRNA expression in neoplasia. Upon using molecular profiling methods such as bead-based flow cytometry, real-time PCR or miRAGE (SAGE analysis) it became possible to determine tissue-specific "signatures" for miRNAs. In line with this, novel molecular classifications of tumors based on their miRNA expression, have provided a wealth of new resources for predictive and prognostic biomarkers for clinical applications in cancer.

\section{Association Between SNPS at MicroRNA Binding Sites and the Risk of Cancer}

When a sequence polymorphism is present within a miRNA transcript it is called a miR-SNP. These miR-SNPs are single-base polymorphisms (SNPs) in the miRNA sequences and are considered to be an important new class of functional polymorphisms in the human genome. Since the mode of action of miRNA is highly sequence-dependent, changing a single base in a miRNA sequence that alters binding specificities may affect multiple genes, thus impacting on one or several biological pathways.

The function of miRNAs may be altered through variations in their own sequence (miR-SNPs) or in their target sequences (called "miR-TS-SNPs") (Figure 3). Since a single miRNA can have multiple mRNA target sites, sequence polymorphisms in general have deeper and more extensive effects from a biological point of view than would sequence alterations to mRNA (Sun et al., 2009). Duan and Pak (2007) identified a SNP within an essential region of miR125 that significantly changed the miR-125a sequence and abolished recognition of its target site. Functional experi- 


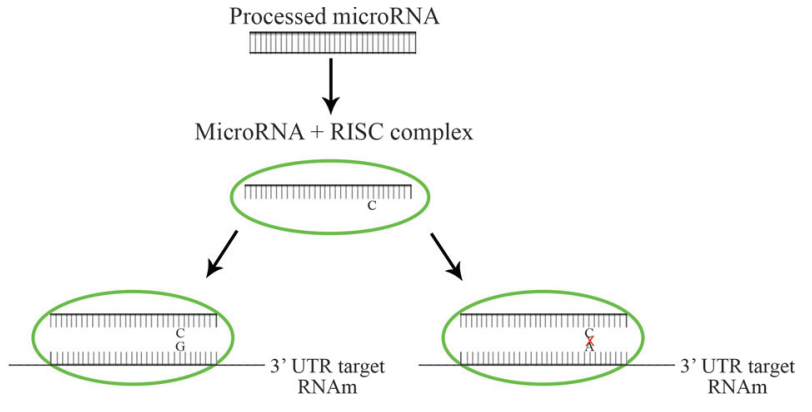

Figure 3 - Possible mechanism for SNP within 3'UTR region of the messenger RNA (mRNA) target gene.

ments in vivo confirmed that the presence of this polymorphism blocked the maturation of miR-125a. Another example of this process is a SNP in the precursor of miR-K5, that is encoded by the human herpes virus in association with Kaposi's sarcoma. This polymorphism correlated with abnormalities in the miRNA cleavage processing by the Drosha enzyme. Similarly, Yang et al (2008) analyzed 41 potentially functional polymorphisms in miRNAs, premicroRNAs and pri-microRNAs that predisposed to bladder cancer (Yang et al., 2008) and found a SNP in GEMIN3 gene and a common haplotype in GEMIN4 that showed significant associations with higher risk of bladder cancer. Moreover, it could be demonstrated that combinations of certain genotypes were strongly associated with predisposition towards bladder cancer, and that the presence of these specific miRNA genotypes could be used as a tool for predicting the risk of developing such tumors. These examples illustrate that variations in the different biogenesis routes for miRNAs affect their own function and consequently the expression of the target messenger RNA.

One of the first epidemiological studies showing a relationship between polymorphisms in the binding region of miRNAs and cancer was published by Landi et al (2008), wherein a relationship between the allele variants of $C D 86$ (polymorphism rs17281995, C > G) and the microRNAs miR-337, miR-582, miR-200a, miR-184 and miR-212 was found to lead to a higher risk of colorectal cancer. It was also shown that there is a relationship between the presence of polymorphism rs 1051690 in the insulin receptor (INSR) and a higher risk of colorectal cancer due to modification of the binding affinity of the miRNAs 618 and 612 (Landi et $a l ., 2008)$. Subsequently, a SNP was identified at the target site of let-7 in the 3'UTR region of the KRAS gene (LCS6KRAS) (Trang et al., 2008). The presence of this polymorphism was associated with increased risk of lung cancer among smokers. This allele variant was detected in $20 \%$ of the patients with lung carcinoma (NSCLC - non-small cell lung carcinoma). In asymptomatic individuals, LCS6$K R A S$ was found in $6 \%$ of the sample analyzed $(\mathrm{n}=2433)$. In a case-control study on lung cancer, the presence of this allele was associated with an increased risk of lung cancer (relative risk of 2.3) among individuals with a history of smoking (mean of 820 cigarettes/year). Functional studies demonstrated that the presence of this polymorphism diminished the binding affinity of let-7 to its target site in $K R A S$, and consequently, increased expression of KRAS. Nonetheless, Christensen et al (2009) reported that in head and neck cancer, this same polymorphism (LCS6-KRAS) was not associated with any general increase in cancer risk, but significantly so with reduced survival (Christensen et al., 2009). Amongst the polymorphisms affecting miRNA target sites, Tchatchou et al (2009) analyzed a group of 11 SNPs and found a strong correlation between the variant rs2747648 $(\mathrm{C} / \mathrm{T})$ in the estrogen receptor 1 (ESR1) gene and an increased risk of breast cancer. This risk was shown to be higher for premenopausal women with a positive family history of cancer (Tchatchou et al., 2009). The mode of action was inferred to be due to a lower binding affinity of miR-453 in the presence of the T allele, and concomitant reduced repression of the ESR 1 gene. Since loss of binding of miR-453, led to overexpression of both the ESR1 mRNA and its receptor protein, the breast cancer cancer risk is expected to be increased.

Taken together the regulation and role of miRNAs and the large number of target sites in functionally important genes and associated pathways provides new insights into cancer risk. It is clear that both a single miR-SNP or a specific combination of miRNA gene variants may act together on their target mRNA sites to constitute a new previously unappreciated mechanism for predisposition to cancer.

\section{Association Between Epigenetic Alterations and MicroRNAs in Cancer}

Various epigenetic alterations may take place during tumor development. Recent studies have indicated that miRNA expression can be regulated by different epigenetic mechanisms, including changes in DNA methylation in promoter regions and histone modification (Scott et al., 2006; Esteller, 2008) (Figure 4). This deregulation seems to involve hypermethylated $\mathrm{CpG}$ islands that map close to specific miRNAs. When expression of such a miRNA is affected, any methylation of this area will simultaneously alter the expression of any target mRNA and proteins modulated by the epigenetically modified miRNA (Scott $e t$ al., 2006).

Recent studies involving epigenetic factors and changes to miRNA expression have mostly been restricted to assays on tumor cells. One of the first studies to be published involved miR-127, which acts to repress the tumor-specific expression of the proto-oncogene BCL-6. After treating tumor cells with chromatin modifying agents, miR-127 showed activity in different types of human cancer cell lines, inferring that its inactivation in these cells could involve chromatin-induced epigenetic alterations (Saito et al., 2006). 


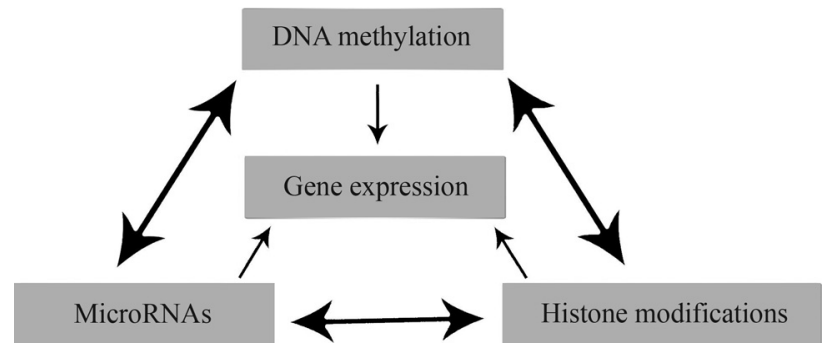

Figure 4 - Crosstalk between genomic methylation, histone modifications and the effects of microRNAs in the gene expression profile.

Suppression of hsa-miR-9-1, hsa-miR-129-2 and hsa-miR-137 in colorectal cancer is, at least partly, mediated by epigenetic mechanisms such as DNA hypermethylation and histone deacetylation, as demonstrated in a recent study by Bandres et al (2009). This study also emphasized that frequent hypermethylation of these miRNA loci in colorectal cancer was correlated with clinicopathological abnormalities. Expression of hsa-miR-9-1 was associated with positive lymph node biopsies in patients with advanced stages of colorectal cancer. DNA methylation was considered to be the most likely mechanism for diminishing or inhibiting the expression of these specific miRNAs. Considering that these miRNAs are not usually expressed in normal mucosal tissue, this epigenetic alteration would diminish the tracking of disease evolution (Bandres et al., 2009).

In breast cancer cell cultures, the homozygous variant of the miRNA hsa-miR-196a-2 (rs11614913, CT) was shown to be significantly associated with diminished risk of breast cancer, and hypermethylation of a $\mathrm{CpG}$ island located 700 base pairs above the precursor region of miR196a-2 led to a reduction in the risk of breast cancer (Hoffman et al., 2009). Based on a series of molecular analyses, these authors suggested that miR-196a-2 might have oncogenic potential in breast cell tumorigenesis, and that functional genetic variations in this miRNA could serve as biomarkers for susceptibility to breast cancer.

Lodygin et al. (2008) reported that miR-34a expression was consistently silenced in different types of cancer by aberrant methylation of $\mathrm{CpG}$ in the promoter region (Lodygin et al., 2008). It was shown that $79.1 \%$ of primary prostate carcinomas had $\mathrm{CpG}$ methylation and concomitant loss of miR-34a expression. Similar observations with differing proportions were made in carcinoma cells in breast (25\%), lung (29.1\%), colon (13\%), kidney (21.4\%) and pancreas tissue $(15.7 \%)$, and in melanomas (43.2\%) and primary melanomas $(62.5 \%)$.

\section{Preservation, Expression and Localization of MicroRNAs in Paraffinized Tissue Samples}

Several methods for evaluating miRNA expression profiles have been implemented, including RT-PCR, microarrays and serial analysis of gene expression (mi-
RAGE). Independent of the approach, success in applying these techniques is essentially limited by the availability of fresh or frozen clinical tissue samples, which are considered to be the most reliable sources of integral RNA (Zhang et al., 2008). Nevertheless, miRNAs turned out to be less affected by fixation in formalin and embedding in paraffin than mRNAs because of their slower degradation, smaller size and lack of a poly-A tail. Good correlations were denoited formiRNA profiles of RNA extracted from frozen samples and those embedded in paraffin (Zhang et al., 2008).

Samples preserved in paraffin are also useful in evaluating the action of a miRNA on a target gene and determining whether it may result in a change in the expression of the corresponding protein. Changes in the expression of proteins regulating the biogenesis of miRNA can also be evaluated at the cellular level. For such analyses, immunohistochemical techniques are useful as these make it possible to detect the location and/or site of subcellular action of the target protein. As an example, the proteins that bind RNA LIN28 and LIN28B prevent precursors of miRNA let-7 from being processed by mature miRNA (Newman et al., 2008). Using immunohistochemistry and tissue microarrays, LIN28 and LIN28B were found to be overexpressed in colon, breast, lung and cervical cancers. Increased expression was associated with physiological repression of let-7 levels and tumor progression, implying a tumorsuppressing role for this miRNA (Viswanathan et al., 2009). In addition, the role of miRNA in tumor tissue samples can be evaluated by means of in situ hybridization (ISH) tests to measure expression levels of specific miRNAs in target cells. Recently, a highly sensitive technique was described for detecting single miRNA molecules in individual cells (Lu and Tsourkas, 2009). The method known as LNA-ELF-FISH employs oligonucleotides from locked nucleic acids with fluorescence for signal amplification, thus allowing miRNAs to be spatially located and quantified inside cells.

In a study on gliomas, and especially multiform glioblastomas, the miRNAs regulated by Dicer, miR-222 and miR-339 were identified using ISH, while the endonuclease and the intercellular adhesion molecule ICAM-1 were evaluated by means of immunohistochemistry. These miRNAs were shown to be expressed by the tumors and negatively regulated ICAM-1, given that the expression of these molecules presented inverse associations in the tissue samples (Ueda et al., 2009). In expression microarrays, there was no difference in Dicer expression between normal prostatic tissue and organ-confined prostate cancer. However, immunohistochemical analysis demonstrated that in normal tissues, Dicer immunoreactivity was detected only in basal cells, proliferative neoplastic cells, and in invasive cancer. The redistribution of Dicer among the cell types seemed to be biologically significant with cancer progression and metastasis, and the level of this endo- 
nuclease continued to increase in the abnormal cells (Chiosea et al., 2009).

The miR-21 is one of the most-studied miRNAs in cancer cases and it is highly expressed in breast cancer. In a cohort analysis using ISH, a progressive increase in the percentage of patient tumors positive for miR-21 was observed, from normal breast tissue (13\%), flat epithelial atypia $(47 \%)$, ductal carcinoma in situ $(75 \%)$ to invasive ductal carcinoma (88\%). In addition, the expression of miR-21 target genes such as PTEN, PDCD4 and TM1 was evaluated in the same tumor samples from the cohort at cellular level, and the cell transformation suppressor TM1 was confirmed as a target of miR-21 in breast tumors, presenting reduced tissue immunoreactivity with progressive lesions, i.e. an inverse relationship with the marker pattern of miR-21 (Qi et al., 2009).

Despite the fast advances in comprehending the biogenesis and action mechanism of miRNAs, many questions regarding their function and influence on central signaling pathways and cell cycle control remain. The complex stochastic nature of gene expression in mammalian cells has wide-ranging impact on phenotypic diversity. It is therefore likely that evaluating mean miRNA expression levels in mixtures of cell populations may result in loss of crucial information for linking miRNA expression to cellular functions. Thus, the physiological role of miRNA in single cells should be more informative in distinguishing the impact of miRNA on signaling networks and cellular pathways relevant to disease (Lu and Tsourkas, 2009).

\section{Gene Therapy and MicroRNAs}

Recently, a new technology of directed artificial-site miRNAs (templates), for increasing or inhibiting endogenous miRNA regulation was describes (Brown et al., 2009). This strategy has been used to detect site-specific target genes in cells, in relation to stem cell therapies and studies on transgenic animals. Through this highly specific new approach a promising strategy has emerged, combining gene therapy with miRNA templates (viruses carrying the target sequence), in an attempt to fully or partially inhibit the expression of these miRNAs.

As described earlier, miRNAs can exert their effects either by acting as tumor suppressors or through favoring cancer development (oncomirs). When hyperexpression of miRNAs contributes towards oncogenesis, the rational strategy is to reduce their expression. In this regard, inhibition of specific endogenous miRNAs has been used through administration of antisense synthetic oligonucleotides, which are complementary to endogenous mature miRNAs.

Oligonucleotide-modified anti-miRNAs (OMAs), also known as 'antagomirs', currently constitute the majority of miRNA inhibition tools (Soifer et al., 2007). Basically three different types of OMAs are used for inhibiting miRNAs, these being oligonucleotides with modifications to the 2-OH group of the ribose residues that involve replacement with 2'-O-methyl (2'-OMe), 2'-Omethoxy-ethyl (2'-MOE) or locked nucleic acid (LNA). These modifications have been incorporated through knowledge gained from interference RNA techniques (RNAi), and were essential for offering resistance to enzymatic degradation, thereby improving OMA stability when exposed to the large quantities of nucleases present in blood and the cell environment. Another important structural change incorporated into these oligonucleotides, with a view to improving their pharmacokinetic properties (such as plasma half-life) and increasing the uptake of the molecule by cells, was the introduction of a cholesterol molecule in the 3' terminal region of the nucleic acid (Bijsterbosch et al., 2000).

Applications of oligonucleotides that specifically inhibit oncomirs, such as mir-21, have been demonstrated in cultures on glioblastoma cells and breast cancer cells, thereby promoting increased caspase activation and mediating apoptosis in these cells (Chan et al., 2005; Si et al., 2007). Furthermore, suppression of mir- 21 gave rise to significant reductions in invasions and lung metastases in cultures on MDA-MB231 breast cancer cells (Zhu et al., 2008).

The efficacy and significance of several OMAs have been examined and validated in several pioneering studies using a model that eliminates miR-122, which is hyperexpressed in rat livers after administration of specifically developed OMAs (Krutzfeldt et al., 2005). These authors were the first to demonstrate nontoxic long-duration silencing generated through intravenous injection of 'antagomirs' (2'-OMe) that were complementary to miR-122, in mice.

Notwithstanding, one of the major obstacles to applying 'antagomirs' in clinical screening is achieving effective release of RNAi in the target tissue (Soifer et al., 2007). Strategies for overcoming these problems have been developed, for example complexation or covalent bonding of lipids and/or proteins released in small RNA molecules (Dykxhoorn et al., 2006). Other alternatives, such as the use of cationic liposomes and cholesterol, conjugation with RNA-packaging phages and RNA aptamers that bind to receptors have been developed to release small RNAs in target cells (Soutschek et al., 2004). Research on local release of biomolecules should substantially improve the therapeutic opportunities for using miRNAs as targets.

Recently, a new form of miRNAs inhibitors called "miRNA sponges" were developed which may be transitorily expressed in mammal cell cultures (Ebert et al., 2007). These "miRNA sponges" are transcripts that are under the control of strong gene promoters (RNA polymerase II) containing tandemly arranged multiple sites for binding to miRNAs of interest and are capable of inhibiting miRNAs as strongly as OMAs (Ebert et al., 2007). 
For miRNAs that present reduced expression in cancer cases, restoration of miRNA levels in the diseased tissue should provide a therapeutic benefit through replacement of the target gene regulation. Introduction of double-stranded miRNAs, which are equivalent to the products from endogenous Dicer and analogous in structure to a siRNA (small interference RNA), may provide transient restoration for underexpressed miRNAs. However, in vivo application of double-strand mimetic miRNA, resembling siRNA, still needs to be evaluated.

To achieve greater persistence of miRNA replacement, a transgenic approach is needed so that expression of specific miRNAs may be induced, starting from a plasmid or viral vector containing the promoters for both the polymerases (II or III) that control the expression of a short hairpin RNA, which is processed subsequent to the mature miRNA.

At present only few studies on the use of miRNAs for in vivo cancer therapy have been published. Gene therapy based on RNAi has been greatly used over recent years. Systemic release of siRNA /shRNA (short hairpin RNA) with an anti-cancer focus employed liposomes, polymers and nanoparticles. Similar strategies and technologies used for siRNA release in cells may also be used with miRNAs.

\section{Conclusion}

miRNAs have emerged over recent years as new regulatory components of the complex mechanisms of gene expression, with implications for many diseases, including cancer. Emerging evidence increasingly demonstrates that miRNAs may also affect or be affected by genetic and epigenetic mechanisms. In addition, miRNAs and miRSNPs are powerful tools for studying disease prognoses and, in the near future, hold tremendous therapeutic promise for clinical medicine and for improvements in cancer control and in curing rates.

\section{Acknowledgments}

We thank Nathan Almeida Pinheiro from Barretos Cancer Hospital for assistance with figure generation. The authors wish to thank Jeremy Squire, Department of Pathology and Molecular Medicine at Kingston General Hospital for suggestions in revising this article.

\section{References}

Ambros V (2003) MicroRNA pathways in flies and worms: growth, death, fat, stress and timming. Cell 13:673-6.

Arndt GM, Dossey L, Cullen LM, Lai A, Druker R, Eisbacher M, Zhang C, Tran N, Fan H, Hetzlaff K, et al. (2009) Characterization of global microRNA expression reveals oncogenic potential of miR-145 in metastatic colorectal cancer. BMC Cancer 20:374-391.

Bandres E, Agirre X, Bitarte N, Ramirez N, Zarate R, RomanGomez J, Prosper F and Garcia-Foncillas J (2009) Epi- genetic regulation of microRNA expression in colorectal cancer. Int J Cancer 125:2737-2743.

Bartel D (2004) MicroRNAs: Genomics, biogenesis, mechanism, and function. Cell 116:281-297.

Bijsterbosch MK, Rump ET, De Vrueh RL, Dorland R, van Veghel R, Tivel KL, Biessen EA, van Berkel TJ and Manoharan M (2000) Modulation of plasma protein binding and in vivo liver cell uptake of phosphorothioate oligodeoxynucleotides by cholesterol conjugation. Nucleic Acids Res 28:2717-2725.

Blenkiron C and Miska EA (2007) miRNAs in cancer: approaches, aetiology, diagnostics and therapy. Hum Mol Genet 1:106-113.

Brown BD and Naldini L (2009) Exploiting and antagonizing microRNA regulation for therapeutic and experimental applications. Nat Rev Genet 10:578-585.

Calin GA, Dumitru CD, Shimizu M, Bichi R, Zupo S, Noch E, Aldler H, Rattan S, Keating M, Rai K, et al. (2002) Frequent deletions and down-regulation of micro- RNA genes miR15 and miR16 at 13q14 in chronic lymphocytic leukemia. Proc Natl Acad Sci USA 99:15524-15529.

Calin GA, Liu CG, Sevignani C, Ferracin M, Felli N, Dumitru CD, Shimizu M, Cimmino A, Zupu S, Dono M, et al. (2004a) MicroRNA profiling reveals distinct signatures in B cell chronic lymphocytic leukemias. Proc Natl Acad Sci USA 101:11755-11760.

Calin GA, Sevignani C, Dumitru CD, Hyslop T, Noch E, Yendamuri S, Shimizu M, Rattan S, Bullrich F, Negrini M, et al. (2004b) Human microRNA genes are frequently located at fragile sites and genomic regions involved in cancers. Proc Natl Acad Sci USA 101:2999-3004.

Calin GA, Cimmino A, Fabbri M, Ferracin M, Wojcik SE, Shimizu M, Taccioli C, Zanesi N, Garzon R, Ageilan RI, et al. (2008) MiR-15a and miR-16-1 cluster functions in human leukemia. Proc Natl Acad Sci USA 105:5166-5171.

Chan JA, Krichevsky AM and Kosik KS (2005) MicroRNA-21 is an antiapoptotic factor in human glioblastoma cells. Cancer Res 65:6029-6033.

Chiosea S, Jelezcova E, Chandran U, Acquafondata M, McHale T, Sobol RW and Dhir H (2009) Up-regulation of dicer, a component of the MicroRNA machinery, in prostate adenocarcinoma. Am J Pathol 169:1812-1820.

Christensen BC, Moyer BJ, Avissar M, Ouellet LG, Plaza SL, McClean MD, Marsit CJ and Kelsey KT (2009) A let-7 microRNA-binding site polymorphism in the KRAS 3' UTR is associated with reduced survival in oral cancers. Carcinogenesis 30:1003-1007.

Duan R and Pak C JP (2007) Single nucleotide polymorphism associated with mature miR-125a alters the processing of pri-miRNA. Hum Mol Genet 16:1124-1131.

Dykxhoorn DM and Lieberman J (2006) Running interference: Prospects and obstacles to using small interfering RNAs as small molecule drugs. Annu Rev Biomed Eng 8:377-402.

Ebert MS, Neilson JR and Sharp PA (2007) MicroRNA sponges: Competitive inhibitors of small RNAs in mammalian cells. Nat Methods 4:721-726.

Esteller M (2008) Epigenetics in cancer. N Engl J Med 358:11481159.

Foekens JA, Sieuwerts AM, Smid M, Look MP, de Weerd V, Boersma AWM, Klijn JG, Wiemer EA and Martens JW (2008) Four miRNAs associated with aggressiveness of 
lymph node-negative, estrogen receptor-positive human breast cancer. Proc Natl Acad Sci USA 105:13021-13026.

Garofalo M and Croce CM (2010) microRNAs: Master regulators as potential therapeutics in cancer. Annu Rev Pharmacol Toxicol 51:25-43.

He L, Thomson JM, Hemann MT, Hernando-Monge E, Mu D, Goodson S, Powers S, Cardon-Cardo S, Lowe SW, Hannon GJ, et al. (2005) A microRNA polycistron as a potential human oncogene. Nature 435:828-833.

Hoffman AE, Zheng T, Yi C, Leaderer D, Weidhaas J, Slack F, Zhang Y, Paranjape T and Zhu Y (2009) microRNA miR196a-2 and breast cancer: a genetic and epigenetic association study and functional analysis. Cancer Res 69:59705977.

Johnson SM, Grosshans H, Shingara J, Byrom M, Jarvis R, Cheng A, Labourier E, Reinert KL, Brown D and Slack FJ (2005) RAS is regulated by the let-7 microRNA family. Cell 120:635-647.

Krützfeldt J, Rajewsky N, Braich R, Rajeev KG, Tuschl T, Manoharan M and Stoffel M (2005) Silencing of microRNAs in vivo with 'antagomirs'. Nature 438:685-689.

Landi D, Gemignani F, Naccarati A, Pardini B, Vodicka P, Vodickova L, Novotny J, Forsti A, Hemmink K, Canzian F, et al. (2008) Polymorphisms within micro-RNA-binding sites and risk of sporadic colorectal cancer. Carcinogenesis 29:579584.

Lodygin D, Tarasov V, Epanchintsev A, Berking C, Knyazeva T, Körner H, Knyazev P, Diebold J and Hermeking H (2008) Inactivation of miR-34a by aberrant $\mathrm{CpG}$ methylation in multiple types of cancer. Cell Cycle 7:2591-2600.

Lu J, Getz G, Miska EA, Alvarez-Saavedra E, Lamb J, Peck D, Sweet-Cordero A, Ebert BL, Mark RH, Ferrnando AA, et al. (2005) MicroRNA expression profiles classify human cancers. Nature 435:834-838.

Lu J and Tsoukars A (2009) Imaging individual microRNAs in single mammalian cells in situ. Nucleic Acids Res 37:e100.

Ma L, Teruya-Feldstein J and Weinberg RA (2007) Tumour invasion and metastasis initiated by microRNA-10b in breast cancer. Nature 449:682-688.

Medina PP and Slack FJ (2008) MicroRNAs and cancer: An overview. Cell Cycle 7:2485-2492.

Miyoshi K, Miyoshi T and Siomi H (2010) Many ways to generate microRNA-like small RNAs: Non-canonical pathways for microRNA production. Mol Genet Genomics 284:95-103.

Newman MA, Thomson JM and Hammond SM (2008) Lin-28 interaction with the Let-7 precursor loop mediates regulated microRNA processing. RNA 14:1539-1549.

O'Donnell KA, Wentzel EA, Zeller KI, Dang CV and Mendell JT (2005) c-Myc-regulated microRNAs modulate E2F1 expression. Nature 435:839-843.

Qi L, Bart J, Tan LP, Platteel I, Sluis TVD, Huitema S, Harms G, Ful L, Hollema H and Berg A (2009) Expression of miR-21 and its targets (PTEN, PDCD4, TM1) in flat epithelial atypia of the breast in relation to ductal carcinoma in situ and invasive carcinoma. BMC Cancer 9:163-171.

Saito Y, Liang G, Egger G, Friedman JM, Chuang JC, Coetzee GA and Jones PA (2006) Specific activation of microRNA127 with downregulation of the proto-oncogene BCL6 by chromatin-modifying drugs in human cancer cells. Cancer Cell 9:435-443.

Scott GK, Mattie MD, Berger CE, Benz SC and Benz CC (2006) Rapid alteration of microRNA levels by histone deacetylase inhibition. Cancer Res 66:1277-1281.

Si M, Zhu S, Wu H, Lu Z, Wu F and Mo Y (2007) miR21-mediated tumor growth. Oncogene 26:2799-2803.

Soifer HS, Rossi JJ and Saetrom P (2007) MicroRNAs in disease and potential therapeutic applications. Mol Ther 15:20702079.

Soutschek J, Akinc A, Bramlage B, Charisse K, Constien R, Donoghue M, Elbashir S, Geick A, Hadwiger P, Harborth J, et al. (2004) Therapeutic silencing of an endogenous gene by systemic administration of modified siRNAs. Nature 432:173-178.

Sun G, Yan J, Noltner K, Feng J, Li H, Sarkis DA, Sommer SS and Rossi JJ (2009) SNPs in human miRNA genes affect biogenesis and function. RNA 15:1640-1651.

Tchatchou S, Jung A, Hemminki K, Sutter C, Wappenschmidt B, Bugert $\mathrm{P}$, Weber BH, Niederacher D, Arnold N and VaronMateeva R (2009) A variant affecting a putative miRNA target site in estrogen receptor (ESR) 1 is associated with breast cancer risk in premenopausal women. Carcinogenesis 30:59-64.

Trang P, Weidhaas JB and Slack FJ (2008) MicroRNAs as potential cancer therapeutics. Oncogene 2:S52-S57.

Ueda R, Kohanbash G, Sasaki K, Fujita M, Zhu X, Kastenhuber ER, McDonald HA, Potter DM, Hamiltom RL and Lotze MT (2009) Dicer-regulated microRNAs 222 and 339 promote resistance of cancer cells to cytotoxic T-lymphocytes by down-regulation of ICAM-1. Proc Natl Acad Sci USA 106:10746-10751.

Vandenboom II TG, Li Y, Philip PA and Sarkar FH (2008) MicroRNA and Cancer: Tiny molecules with major implications. Curr Genomics 9:97-109.

Viswanathan SR, Powers JT, Einhorn W, Hoshida Y, Ng TL, Toffanin S, O'Sullivan M, Lu J, Philips LA and Lockhart VL (2009) Lin 28 promotes transformation and is associated with advanced human malignancies. Nat Genet 2009 41:843-848.

Volinia S, Calin GA, Liu C, Ambs S, Cimmino A, Petrocca F, Visone R, Iorio M, Roldo C, Ferracin M, et al. (2006) A microRNA expression signature of human solid tumors defines cancer gene targets. Proc Natl Acad Sci USA 103:2257-2261.

Yang H, Dinney CP, Ye Y, Zhu Y, Grossman HB and Wu X (2008) Evaluation of genetic variants in microRNA-related genes and risk of bladder cancer. Cancer Res 68:2530-2537.

Zhang X, Chen J, Radcliffe T, Lebrun DP, Tron VA and Feilotter H (2008) An array-based analysis of microRNA expression comparing matched frozen and formalin-fixed paraffinembedded human tissue samples. J Mol Diagn 10:513-519.

Zhu S, Wu H, Wu F, Nie D, Sheng S and Mo Y (2008) MicroRNA-21 targets tumor suppressor genes in invasion and metastasis. Cell Res 18:350-359.

Associate Editor: Carlos F.M. Menck

License information: This is an open-access article distributed under the terms of the Creative Commons Attribution License, which permits unrestricted use, distribution, and reproduction in any medium, provided the original work is properly cited. 